\title{
THE USE OF SOCIAL MEDIA MARKETING IN PERSONAL SELLING
}

\section{NUGZAR TODUA}

D.Sc. Professor,

Academician of Georgia Economic Sciences Academy

Ivane Javakhishvili Tbilisi State University, Georgia

nugzar.todua@tsu.ge

\section{BEKA VASHAKIDZE}

\section{PhD Student}

Ivane Javakhishvili Tbilisi State University, Georgia

beka.vashakidze866@eab.tsu.edu.ge

Abstract. The article shows that the global network, Internet, is socializing at a very high speed, and this is one of the main trends in the modern online system. The audience of social media growing rapidly both in the world and in Georgia. This growth was facilitated by the fact that social media went beyond teen entertainment. Social media today is a platform for communication, study and work where people of different generations began to join.

This article explores the importance of social media for marketing. It is noted that nowadays social media marketing has become an important component of online marketing, which has shown its effectiveness for companies targeted at the target audience. Social media marketing applications and platforms drive customer engagement, collaboration, and content sharing. The role of social media marketing in personal sales is also analyzed and the main problems associated with it are identified. With social media technology, sellers have a unique opportunity to build, influence and manage individual relationships with customers. Social media technologies help sellers engage customers through information sharing, interactivity, and problem-solving. Social media is an essential tool for salespeople to use to build customer relationships. However, there are not enough criteria for determining the effectiveness of the use of social networks by sales personnel. In this regard, studying the role of social media marketing in personal sales is one of the most pressing issues. Although some work has been done recently in the area of consumer behavior in Georgia, the issues of consumer behavior concerning personal sales in the Georgian market are still poorly understood and requires proper scientific development. Based on the above, the purpose of this work was to determine the attitude of Georgian consumers to social media marketing activities carried out by personal sellers, for which marketing research was conducted.

The research was carried out in two stages based on qualitative and quantitative research methods. At the first stage, a qualitative study was carried out, which included a focus group and in-depth interview methods. At this stage, hypotheses were formulated and priorities were set for further research. In the second stage, we used the customer survey method, and as a research tool, we chose a questionnaire consisting of several structured questions. The survey was conducted using electronic and personal interview methods. A systematic random sampling method was used. The confidence interval is $95 \%$ and the margin of errors is set to be equal to $4 \%$. The survey was carried out with 1022 respondents aged 18 years and more, which represent $0.03 \%$ of the total population of Georgia. The study area was different cities of Georgia: Tbilisi, Kutaisi, Batumi, Rustavi, Sachkhere, Ambrolauri, Gori, Zugdidi, Senaki, Poti, and Kvareli. Based on this the survey results were analyzed using statistical software SPSS (version 21.0) for windows.

Marketing survey showed that the majority of respondents (70\%) actively use social media for personal sales. In this regard, Facebook is the leader among the most used social media platforms. The survey showed interest, reliability, and satisfaction of the respondents from the information provided by the websites related to personal sales. Marketing research has shown that the social media activity of salespeople is a very important factor for consumers when making a purchasing decision. Based on the analysis of variance, statistically significant values were obtained, which reflect the influence of consumer interest, awareness, trust and satisfaction on social networks associated with personal sales on their purchasing decisions.

\section{KEYWORDS: SOCIAL MEDIA MARKETING, GEORGIAN MARKET, PERSONAL SALES, MARKETING RESEARCH.}

For citation: Todua, N., Vashakidze, B. (2020). The Use of Social Media Marketing in Personal Selling. Globalization and Business, 10. 121-129. https://doi.org/10.35945/gb.2020.10.015 


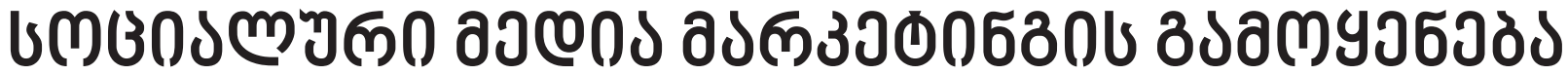

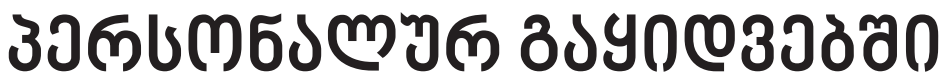

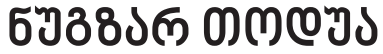

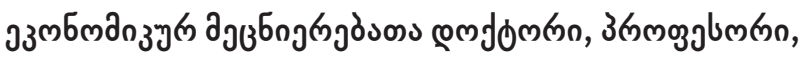

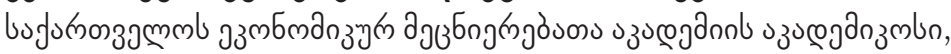

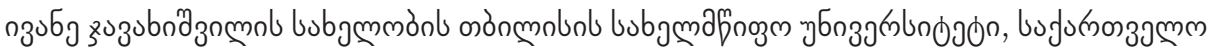

nugzar.todua@tsu.ge

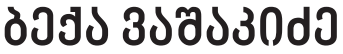

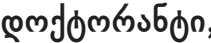

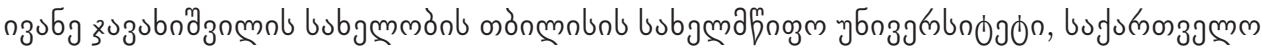

beka.vashakidze866@eab.tsu.edu.ge

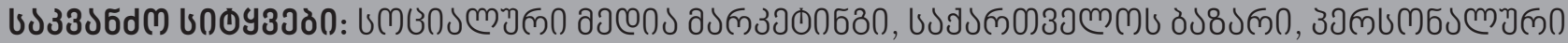

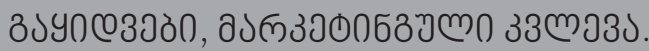

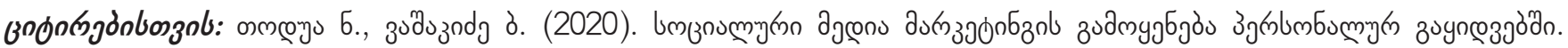

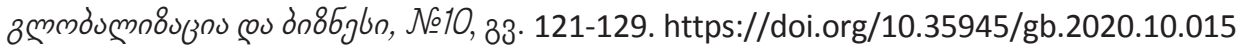

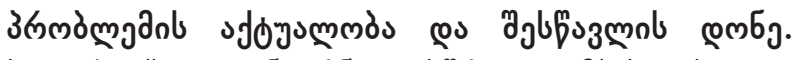

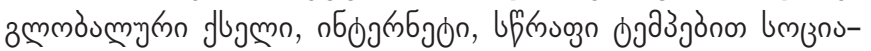

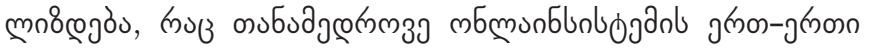

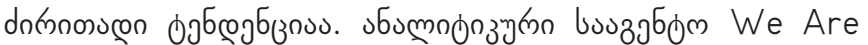

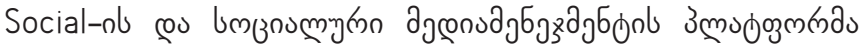

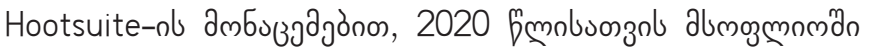

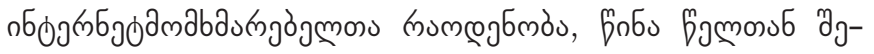

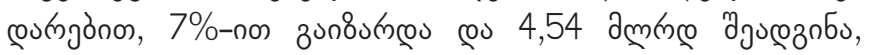

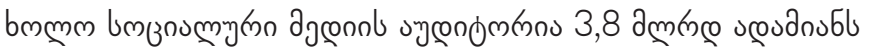

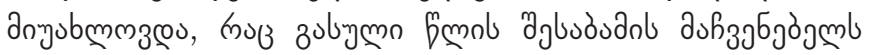
9,2\%-non uģauogò (We Are Social \& Hootsuite, 2020).

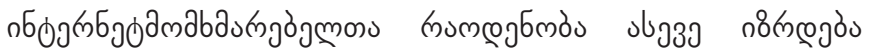
bufurnos замm

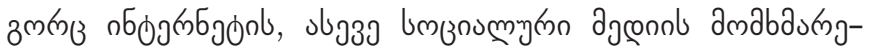

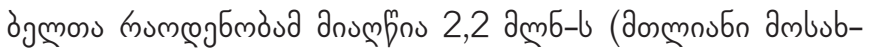

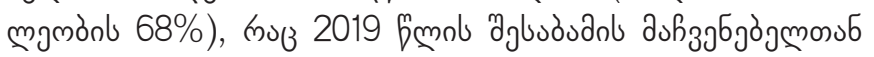

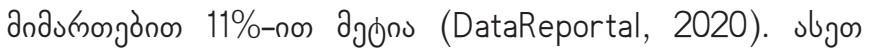

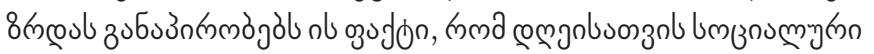

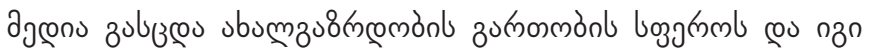

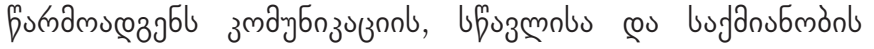

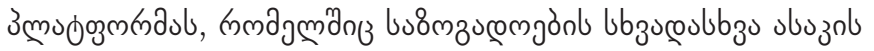

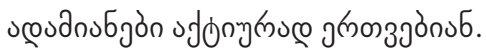

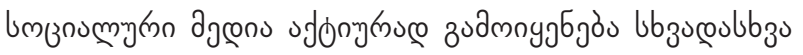

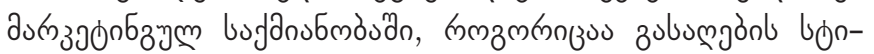

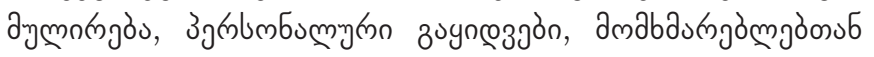

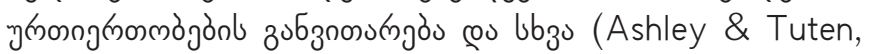

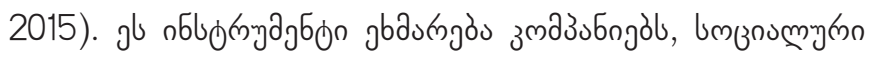

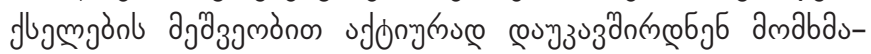

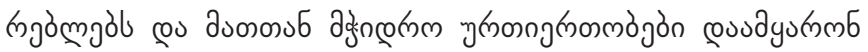

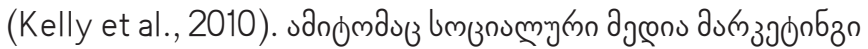

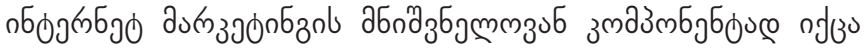

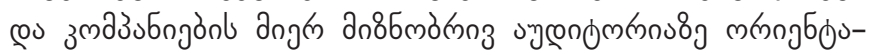

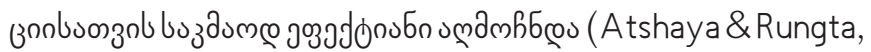

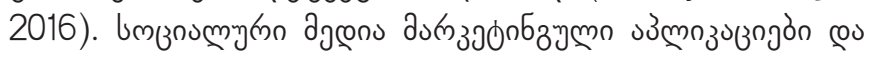

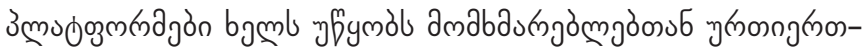

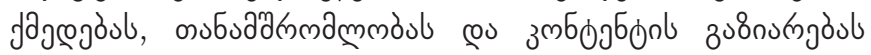

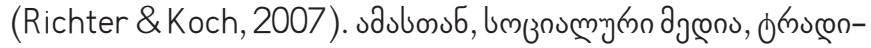

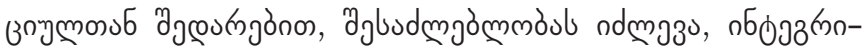

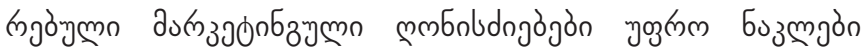

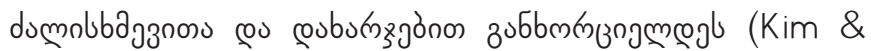

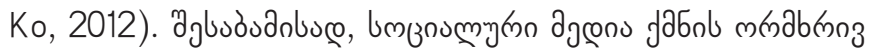
з заз

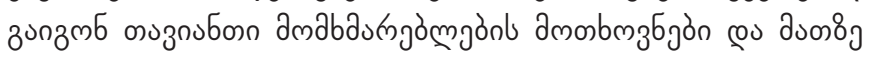

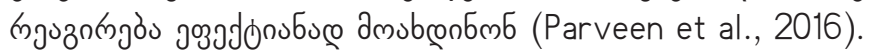

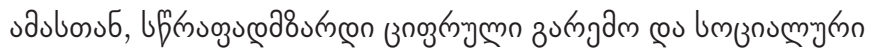

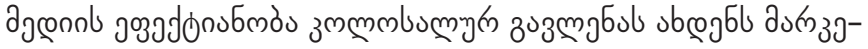

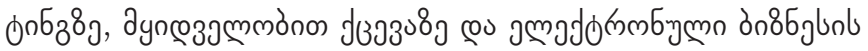
उmodfo 3080 j (Shin et al., 2015).

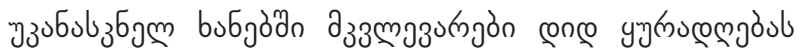

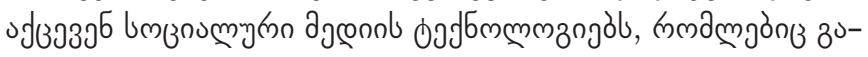

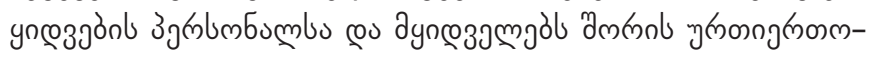

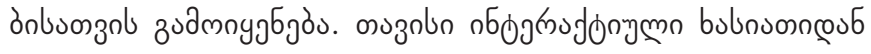




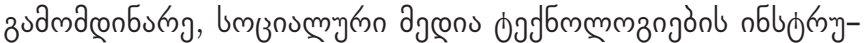

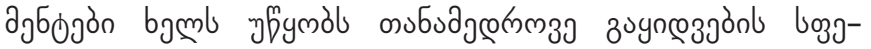

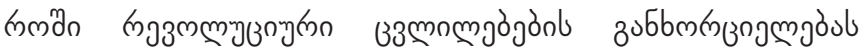

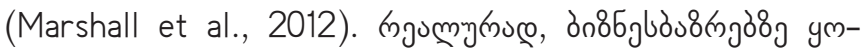

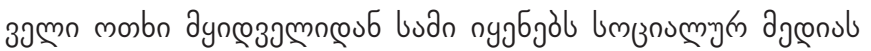

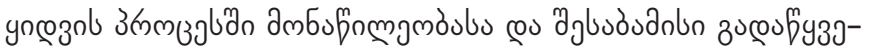
onmgàgonl dnbuggoupe (Minsky \& Quesenberry, 2016). ju

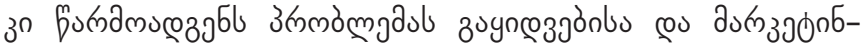
zol bgmad

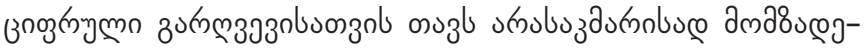

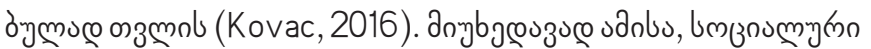

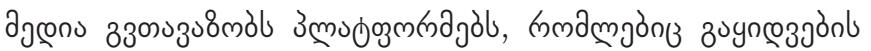

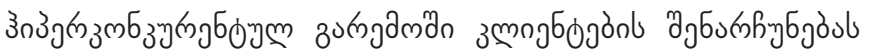

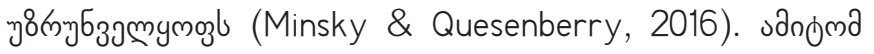

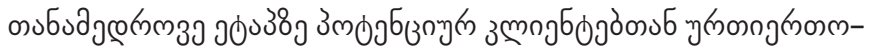

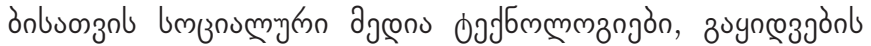

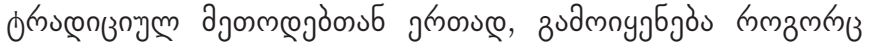

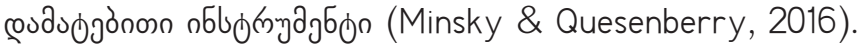

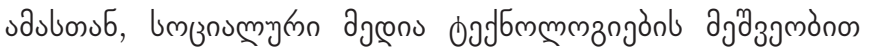

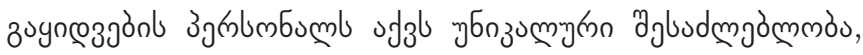

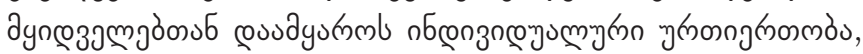

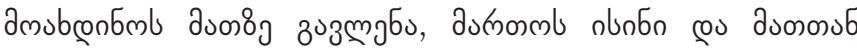

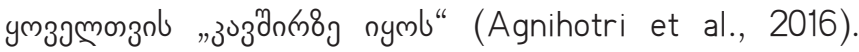

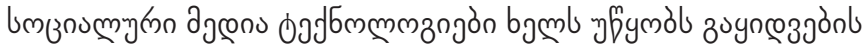

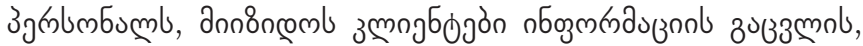

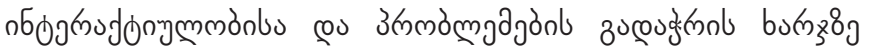
(Hennig-Thurau et al., 2010).

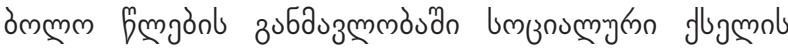

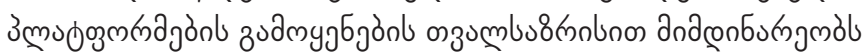

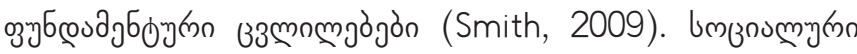

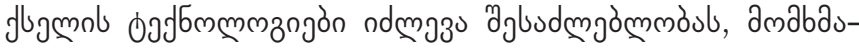

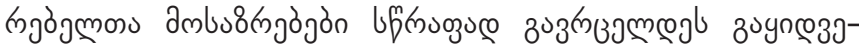

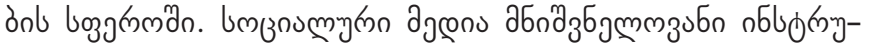

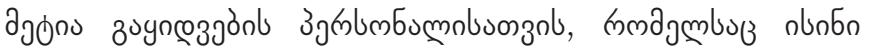

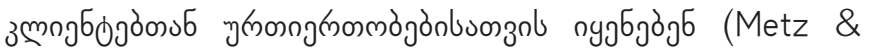

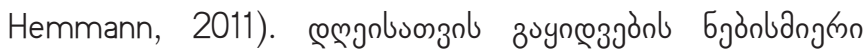

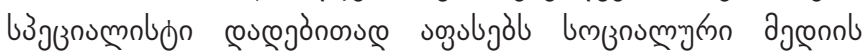

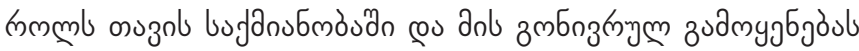

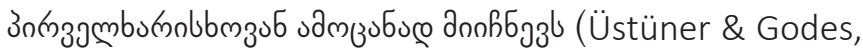

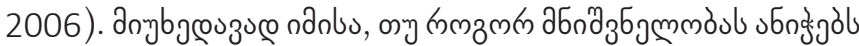

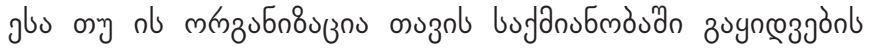

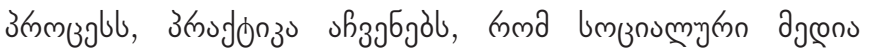

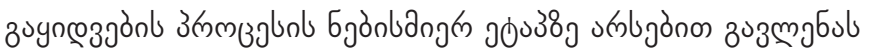

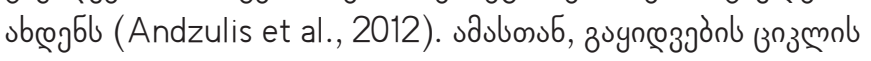

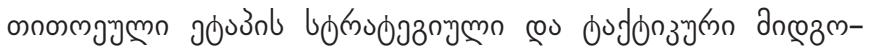

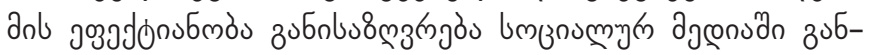

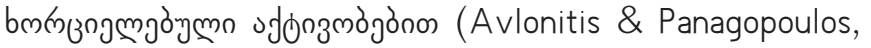

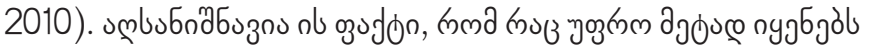

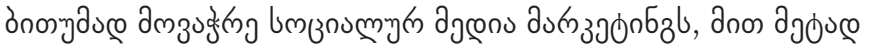

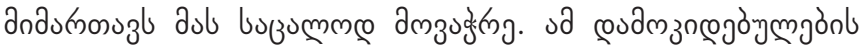

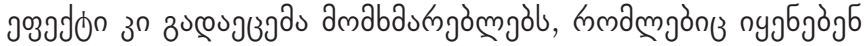

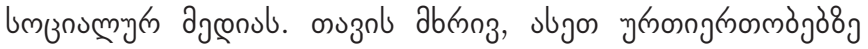
3uzmojbul sbegbl zuyn@

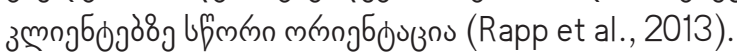

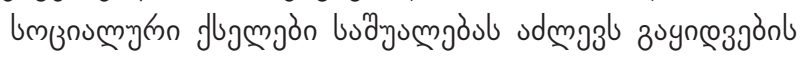

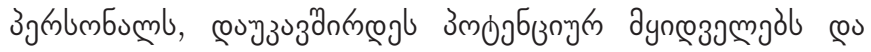

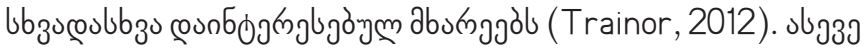

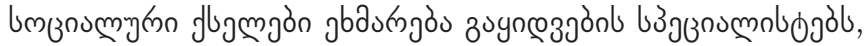

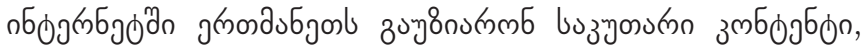

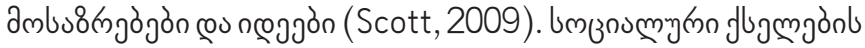

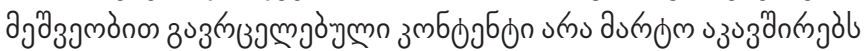

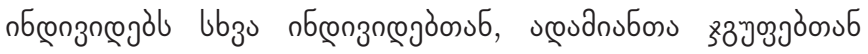

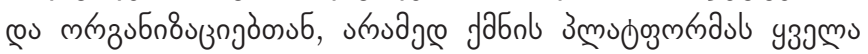

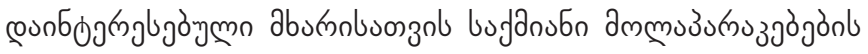
furnasmonzobsonzol (Rodriguez et al., 2012).

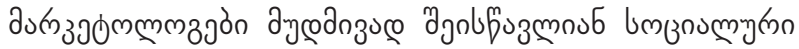

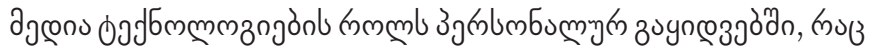

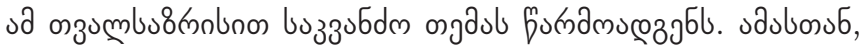

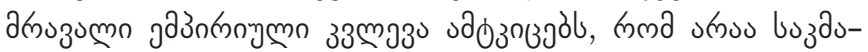

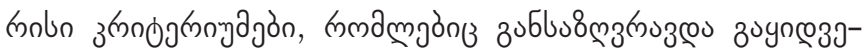

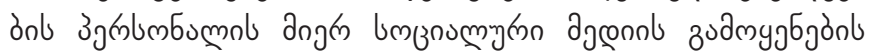

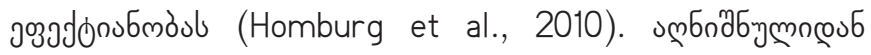

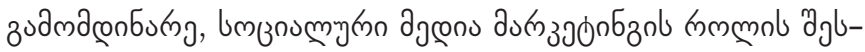

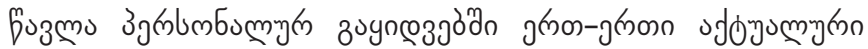

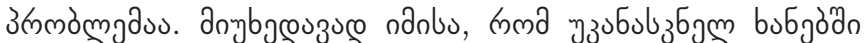

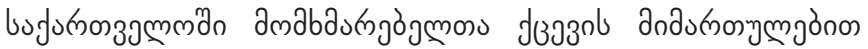

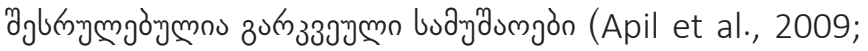
Todua \& Dotchviri, 2015; Todua \& Jashi, 2016; Todua etal., 2016; Mghebrishvili \& Urotadze, 2016; Seturi \& Urotadze, 2017; Todua, 2017a; Todua, 2017b; Todua \& Gogitidze, 2017; Todua \& Jashi, 2018; Todua \& Vashakidze, 2018; Todua, 2019; Todua \& Maghlakelidze, 2019; Urotadze,

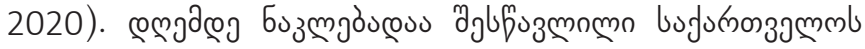

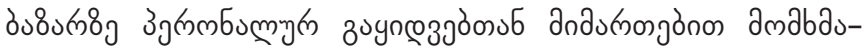

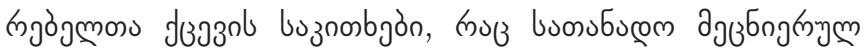

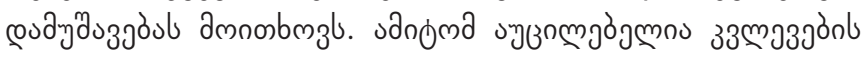

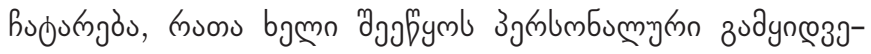

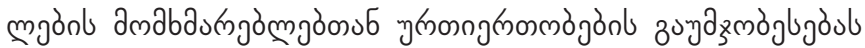

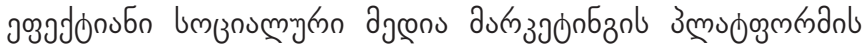

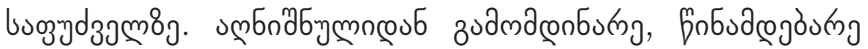

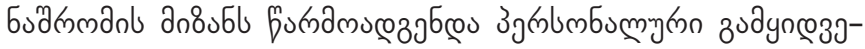

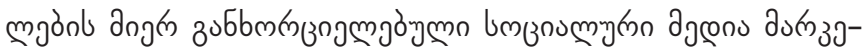

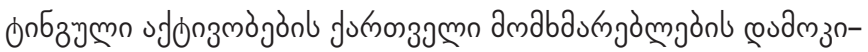

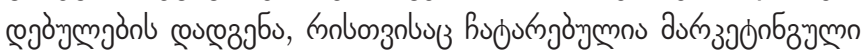
उзलग3०.

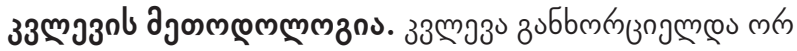

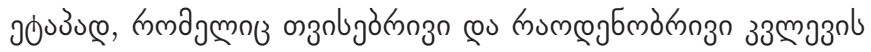

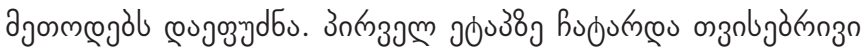

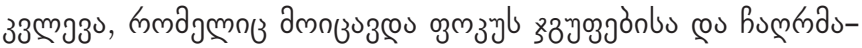

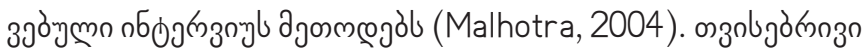




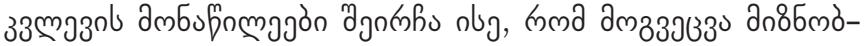

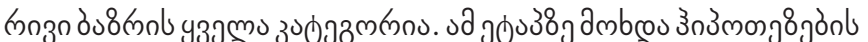

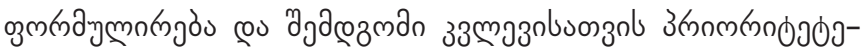

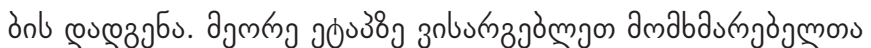

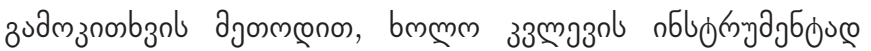

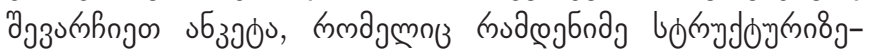

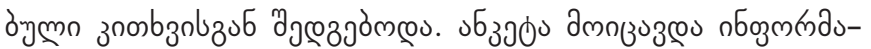

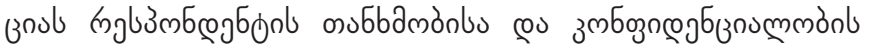

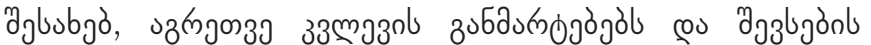

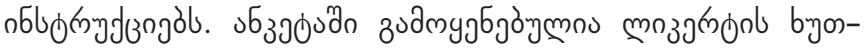

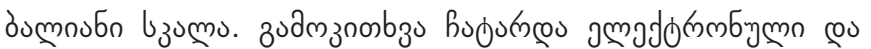

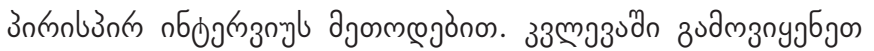

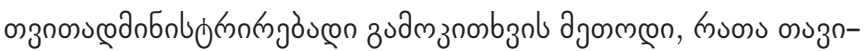

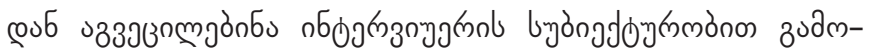

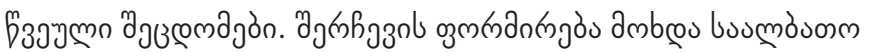

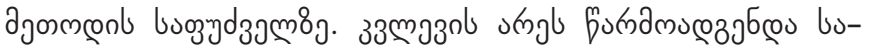

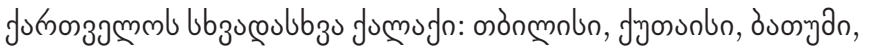

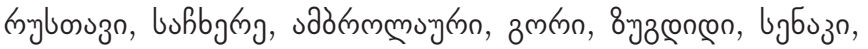

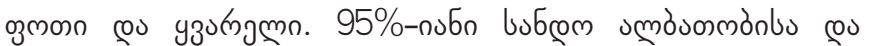

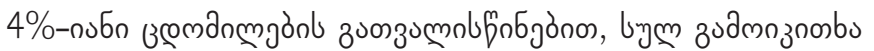

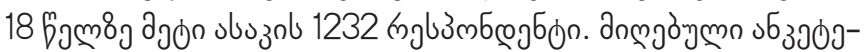

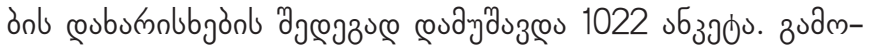

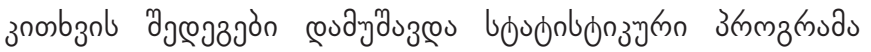

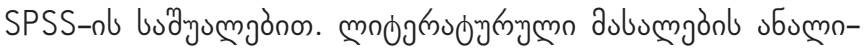

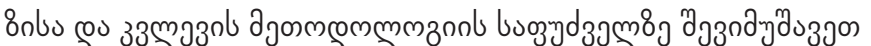

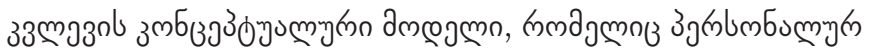

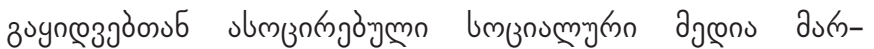

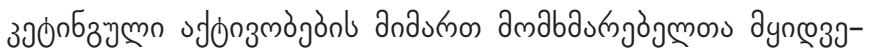

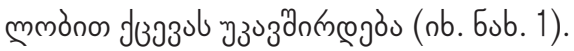

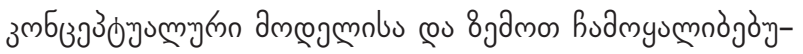

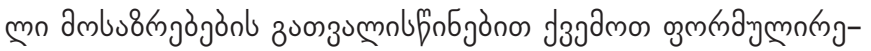

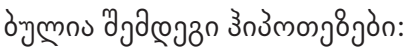

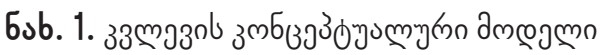

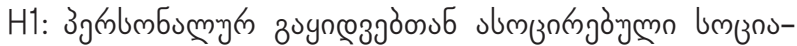

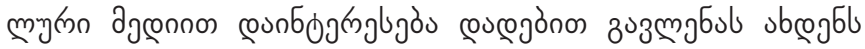

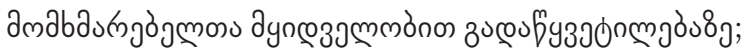

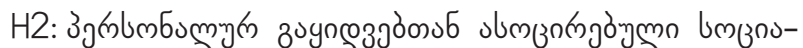

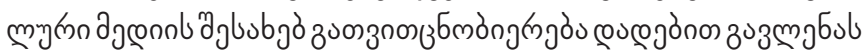

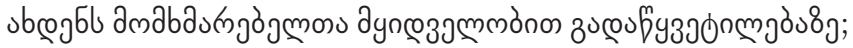

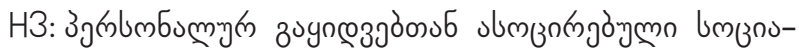

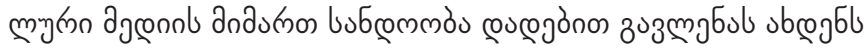

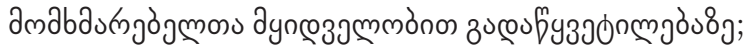

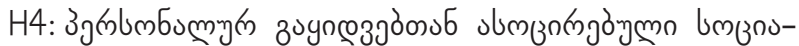

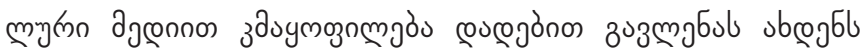

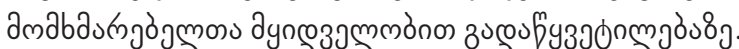

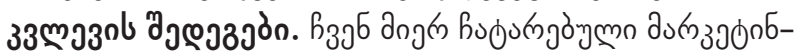

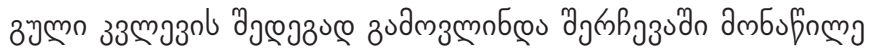

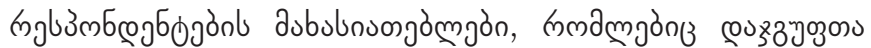

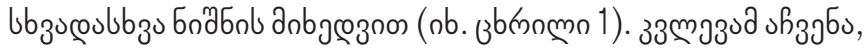

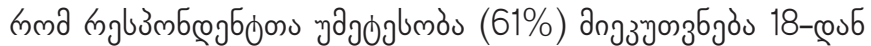

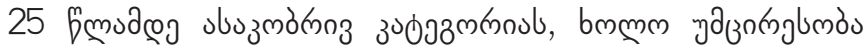

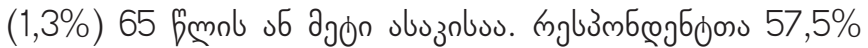
s non jumo gou 42,5\% - auasuzuzn.

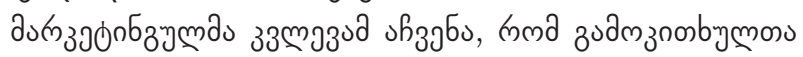

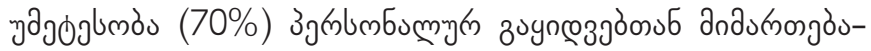

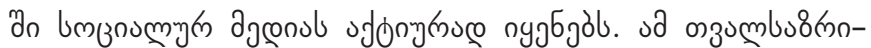

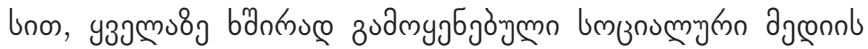

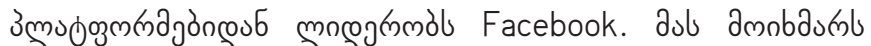

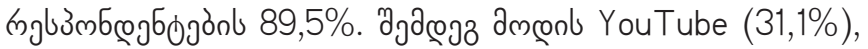
Instagram (16,7\%), Google+ (19,3\%), Linkedln (7,8\%), Twitter (57,2\%) cos MySpace (2,2\%). Ubzu umz̧numynono Jlugmgòn (Pinterest, Tumblr, Flickr, Reddit, Ask.fm,

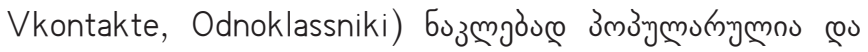

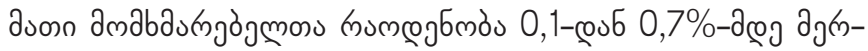

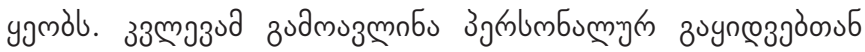

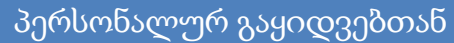

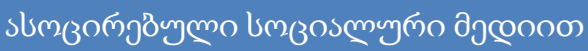

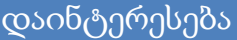

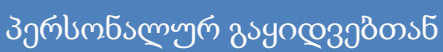

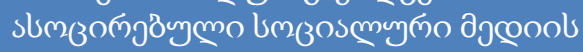

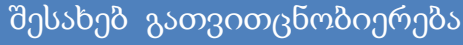

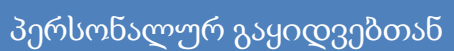

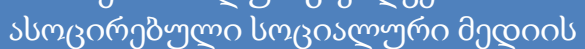

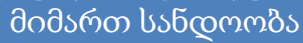

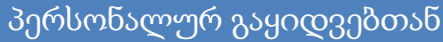

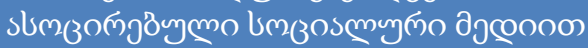
заsумовомјठ̈s

\section{H1}

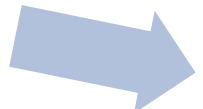

H2

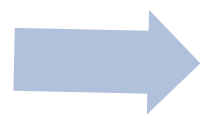

H3
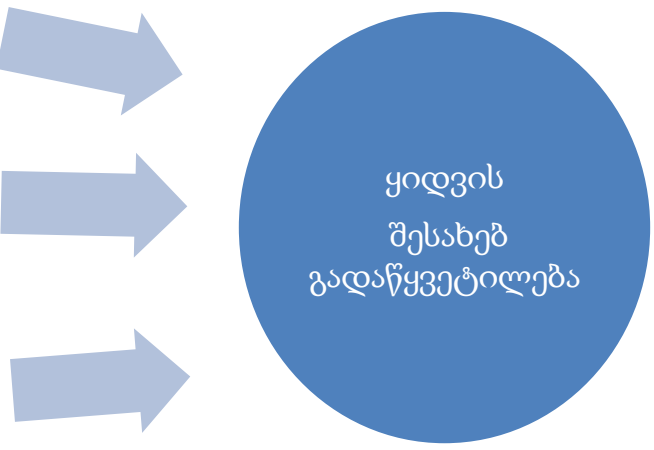

H4

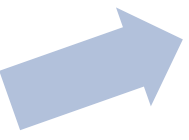




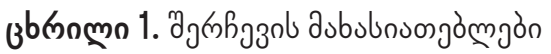

\begin{tabular}{|c|c|c|c|}
\hline 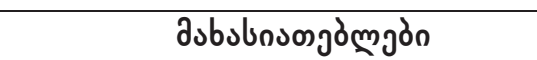 & 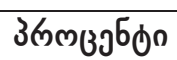 & 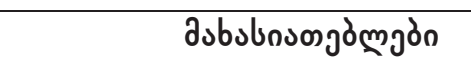 & 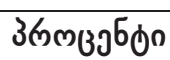 \\
\hline nabozon & & 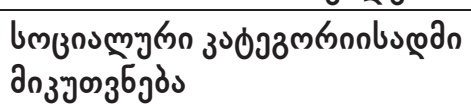 & \\
\hline $18-25$ & 61,0 & 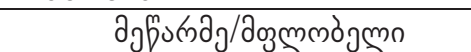 & 2,5 \\
\hline $26-35$ & 21,7 & 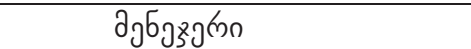 & 6,7 \\
\hline $36-45$ & 6,0 & b3̈gzоumobøo & 26.0 \\
\hline $46-55$ & 6,3 & 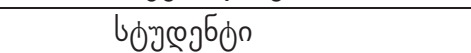 & 32,4 \\
\hline $56-65$ & 3,7 & 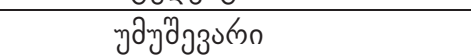 & 24,7 \\
\hline 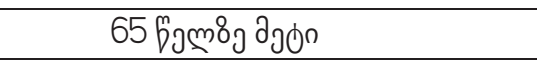 & 1,3 & conobsbmolin & 3,1 \\
\hline bjgbo & & 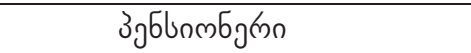 & 4,6 \\
\hline Jumo & 57,5 & 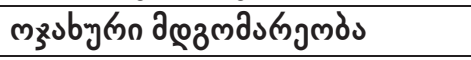 & \\
\hline gงdoz०z० & 42,5 & dundmbgno & 66,8 \\
\hline 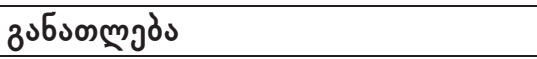 & & coumz̧ubjöyma & 29,0 \\
\hline bodyıलmm & 4,9 & 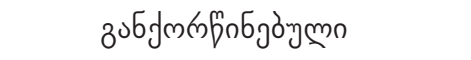 & 2,2 \\
\hline 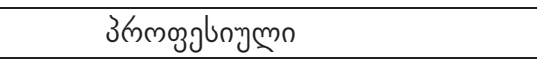 & 5,9 & $d_{3} m \cap 3 n$ & 2,0 \\
\hline 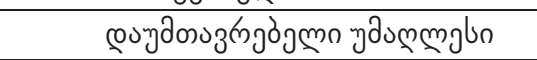 & 35,7 & 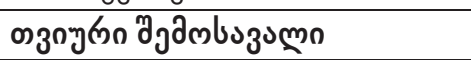 & \\
\hline \multirow{2}{*}{ ydummglon } & \multirow{2}{*}{53,5} & 500 mоmuaøo & 7,1 \\
\hline & & $501-1000$ mumn & 23,0 \\
\hline posustajos & & $1001-1500$ mumn & 19,2 \\
\hline 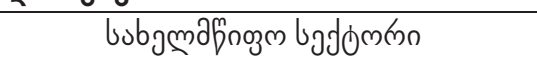 & 13,2 & $1501-2000$ mumn & 14,1 \\
\hline 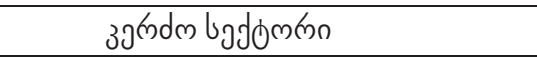 & 45,5 & 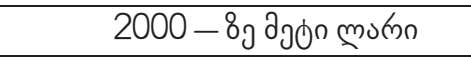 & 17,0 \\
\hline 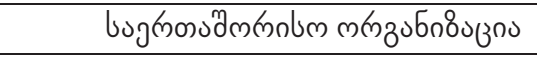 & 2,3 & yumn 3ubyb8g & 19,7 \\
\hline 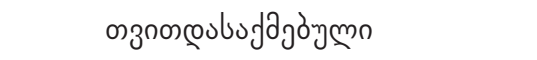 & 4,8 & & \\
\hline 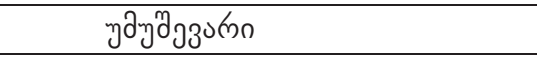 & 24,7 & & \\
\hline bbzo & 9,5 & & \\
\hline
\end{tabular}

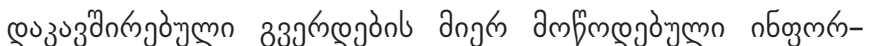

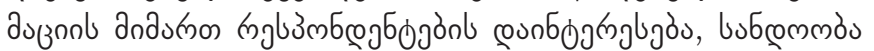
एu zалумоुnm

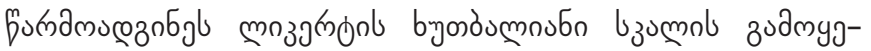

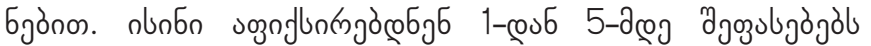

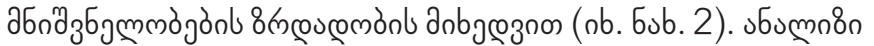

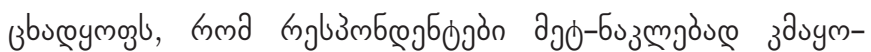

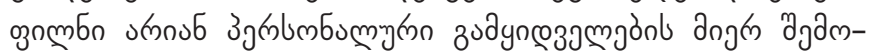

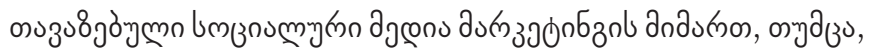

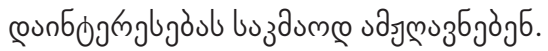

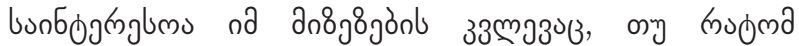

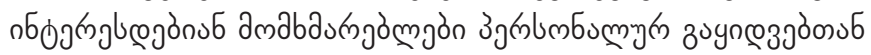

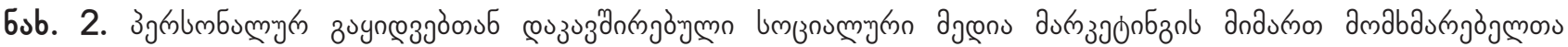

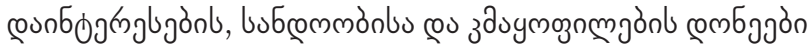

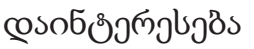

usбcommis

зазумозомудs

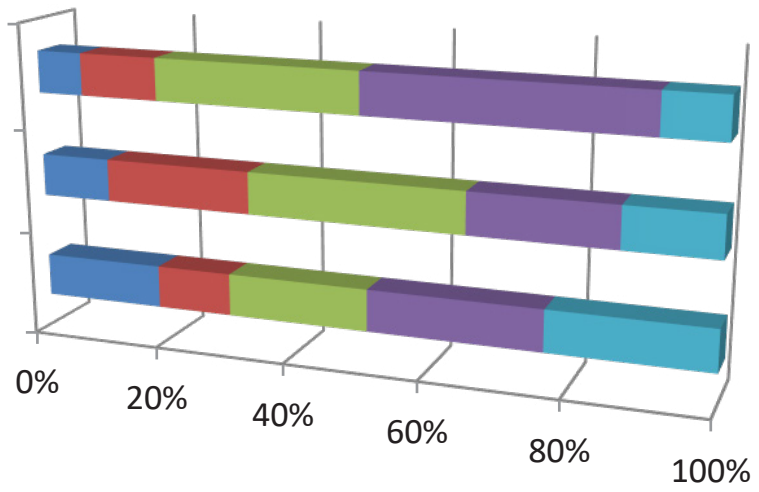

dsmos6 cos 8 smo com6j

- cosissmo gomfj

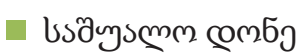

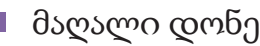

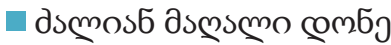


ubmgnn

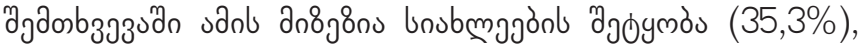

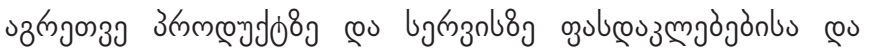

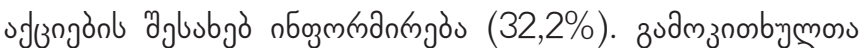

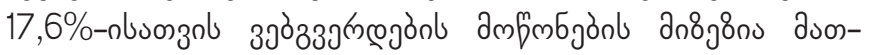

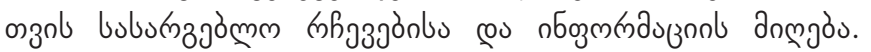

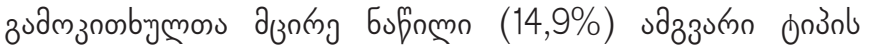
зэ

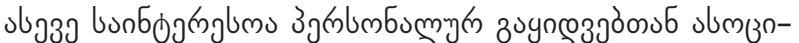

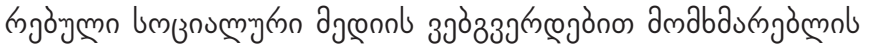

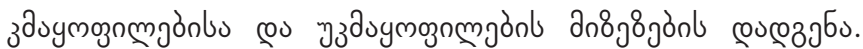

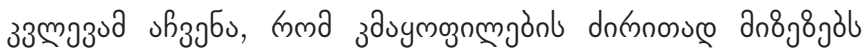

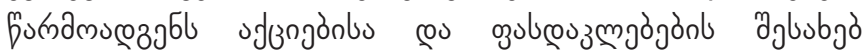

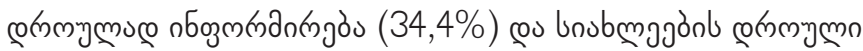

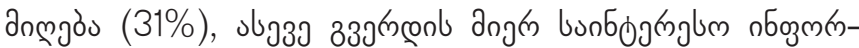
auzonl zudm

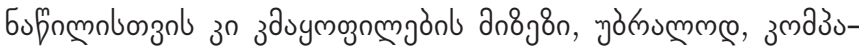

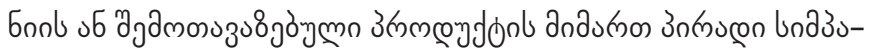

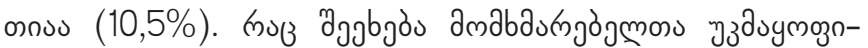

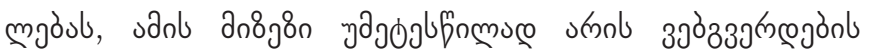

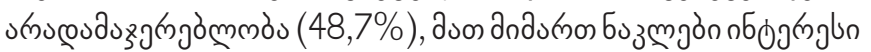

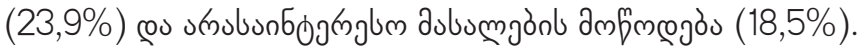

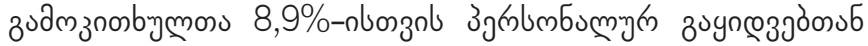

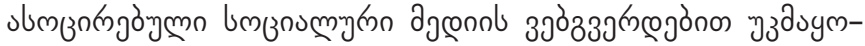

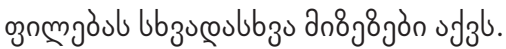

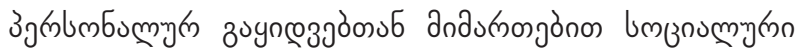

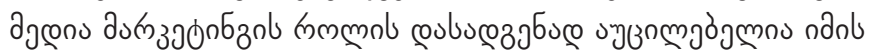

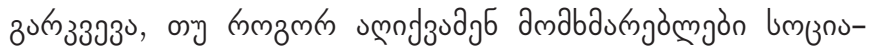

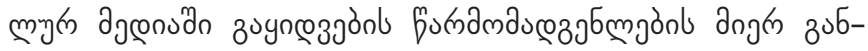

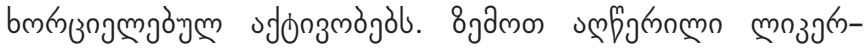

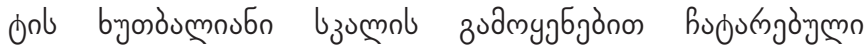

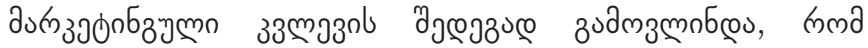

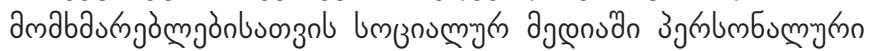

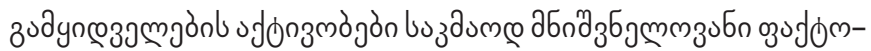

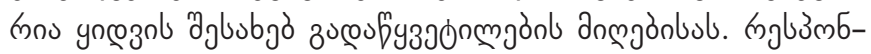

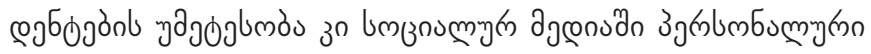

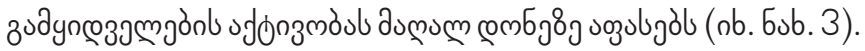

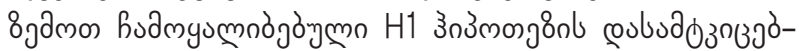

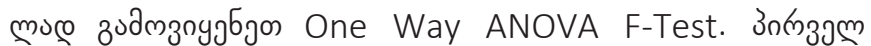

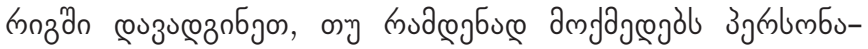

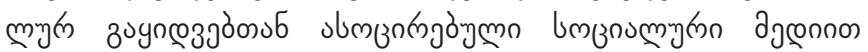

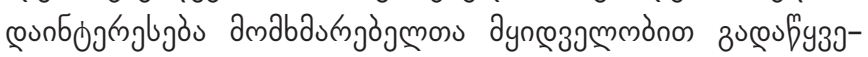
on

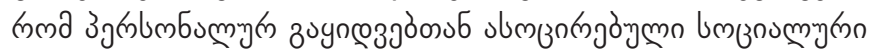

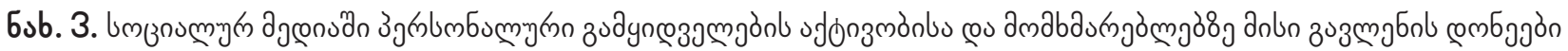

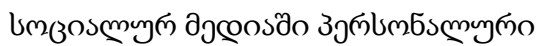

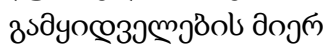

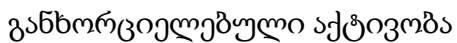

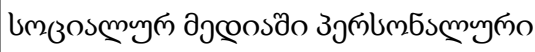
зsауощз

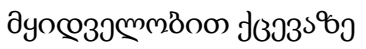

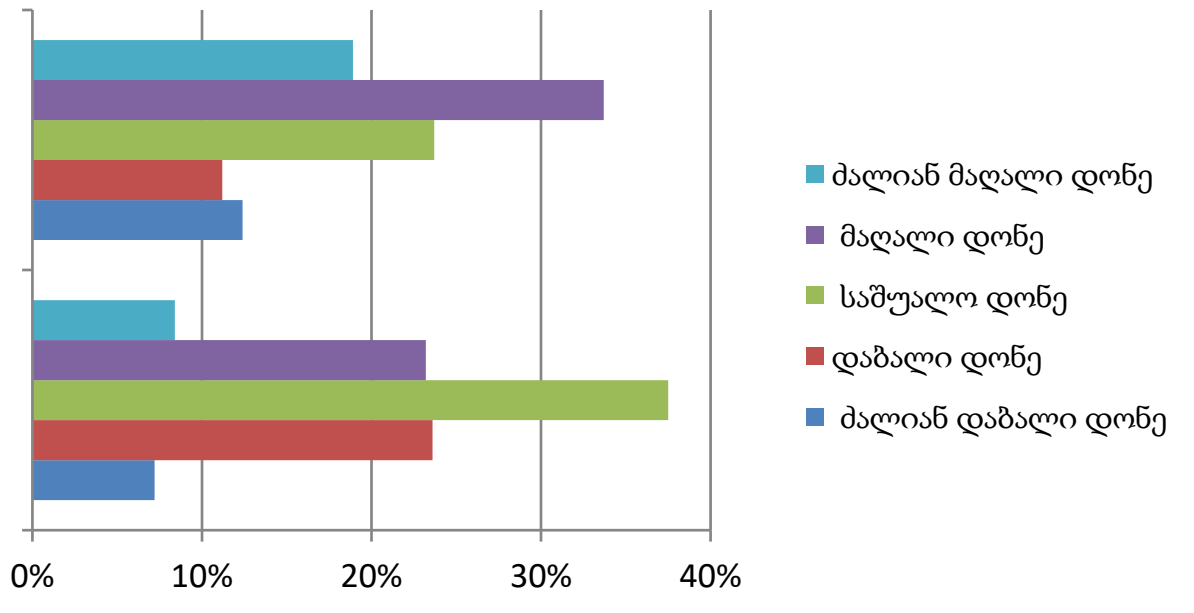

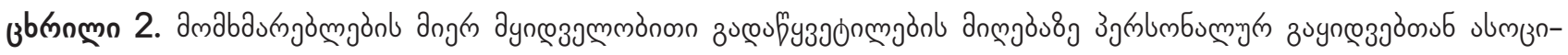

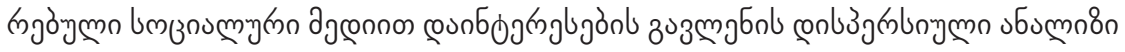

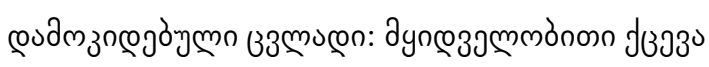

\begin{tabular}{|c|c|c|c|c|c|}
\hline & 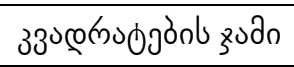 & $\mathrm{df}$ & 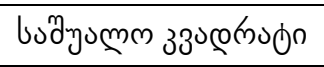 & $\mathrm{F}$ & $P$ \\
\hline 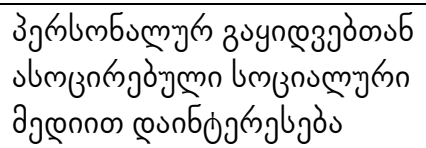 & 7,251 & 4 & 2,061 & 1,871 & ,003 \\
\hline 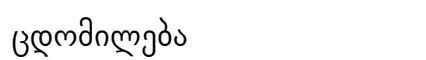 & 767,447 & 518 & 1,081 & & \\
\hline
\end{tabular}




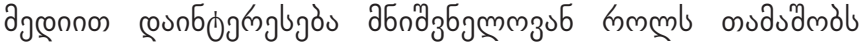

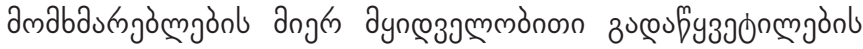

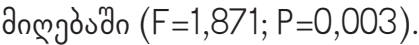

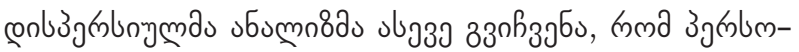

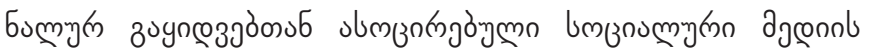

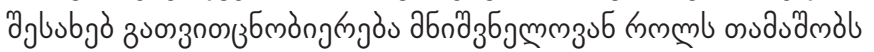

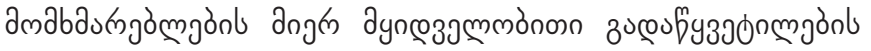

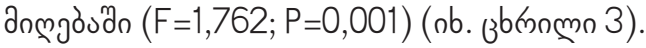

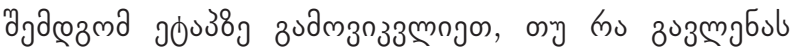

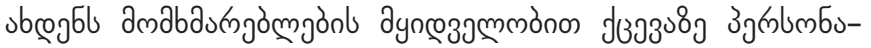

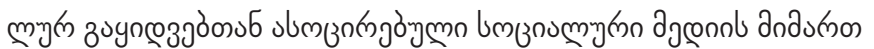

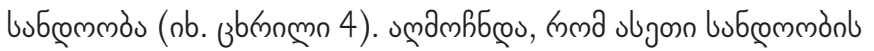

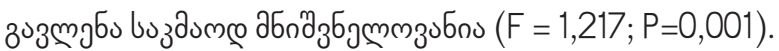

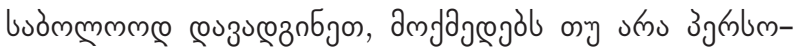

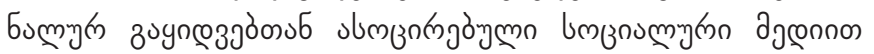

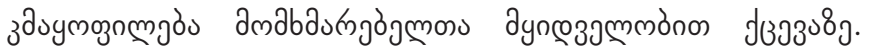

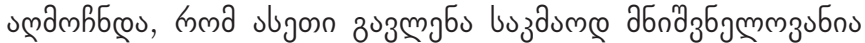

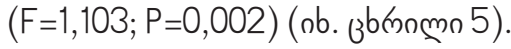

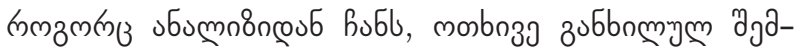

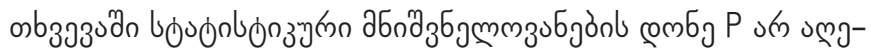

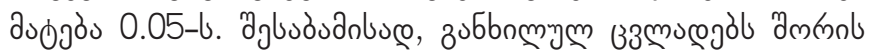

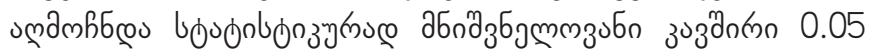

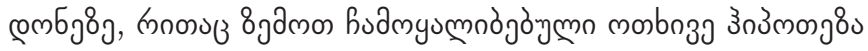
cospojozico.

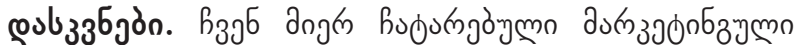

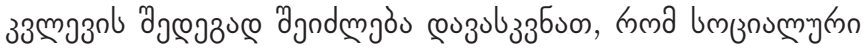

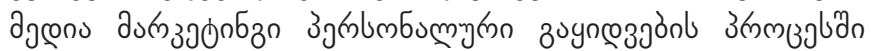

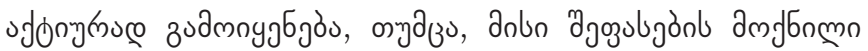

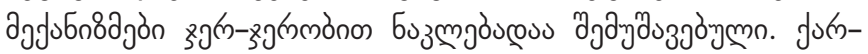

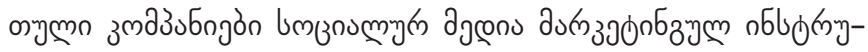

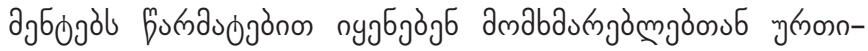

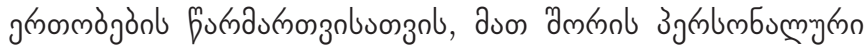

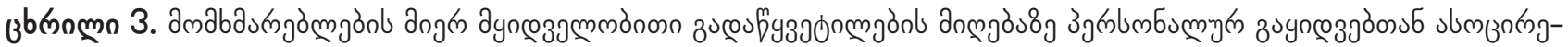

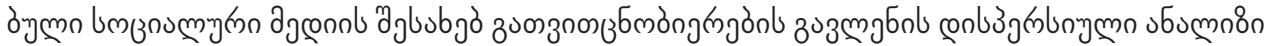

\begin{tabular}{|c|c|c|c|c|c|}
\hline \multicolumn{6}{|c|}{ 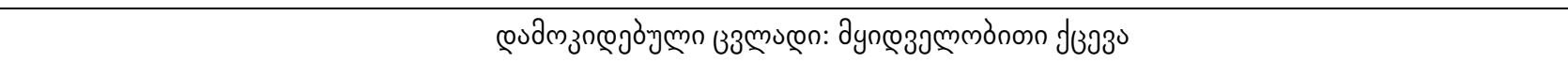 } \\
\hline & 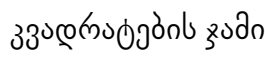 & df & 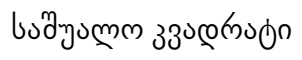 & $\mathrm{F}$ & $P$ \\
\hline 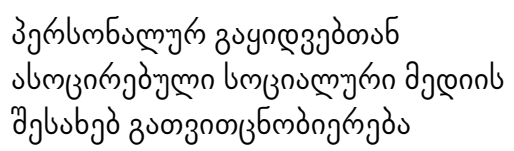 & 8,939 & 3 & 1,087 & 1,762 & ,001 \\
\hline 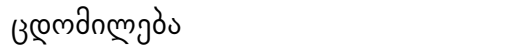 & 767,447 & 518 & 1,081 & & \\
\hline
\end{tabular}

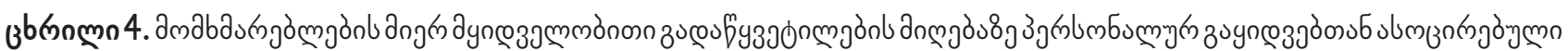

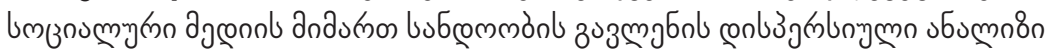

\begin{tabular}{|c|c|c|c|c|c|}
\hline \multicolumn{6}{|c|}{ 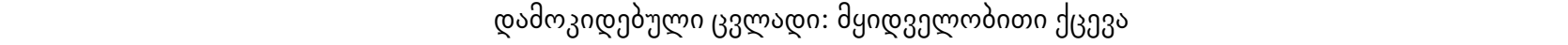 } \\
\hline & 33دم & $\mathrm{df}$ & 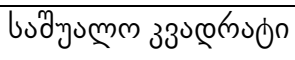 & $\mathrm{F}$ & $P$ \\
\hline 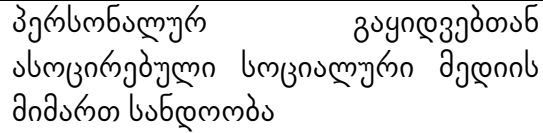 & 7,167 & 4 & 1,534 & 1,217 & 001 \\
\hline उемдnmjòs & 767,447 & 518 & 1,081 & & \\
\hline
\end{tabular}

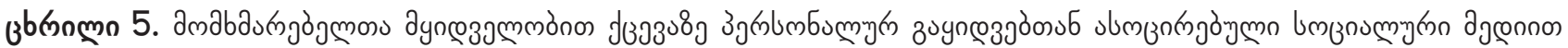

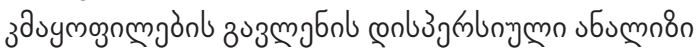

\begin{tabular}{|c|c|c|c|c|c|}
\hline \multicolumn{6}{|c|}{ 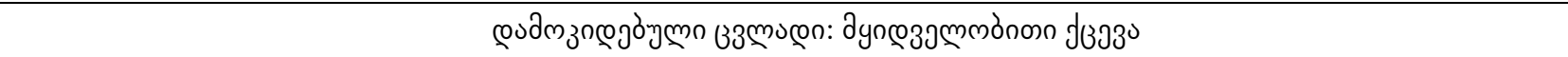 } \\
\hline & 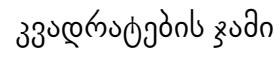 & $d f$ & 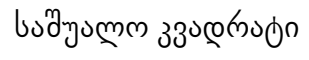 & $\mathrm{F}$ & $\mathrm{p}$ \\
\hline 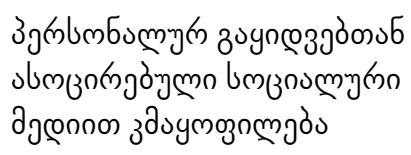 & 6,263 & 5 & 1,351 & 1,103 & ,002 \\
\hline 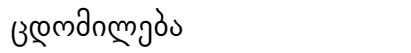 & 767,447 & 518 & 1,081 & & \\
\hline
\end{tabular}




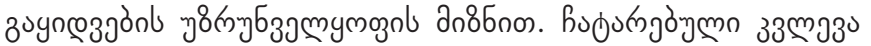

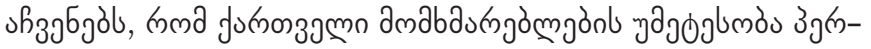

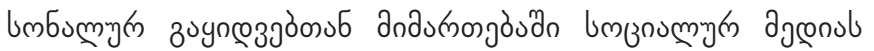

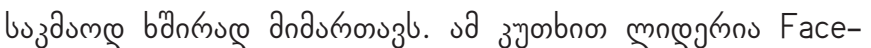

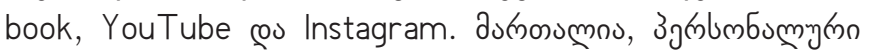

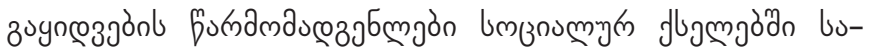

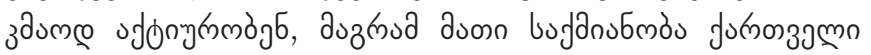

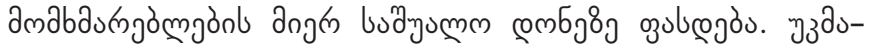

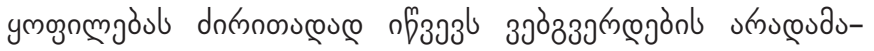

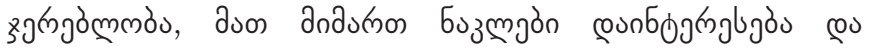

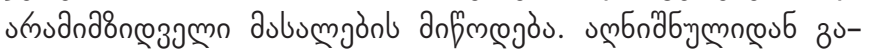

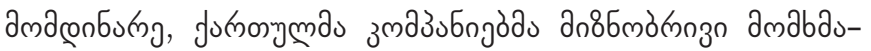

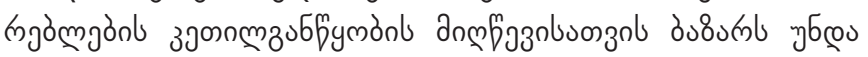

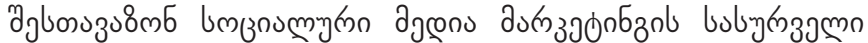

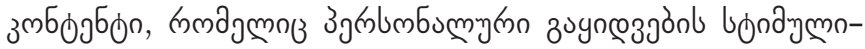
ngòub àyfymal bymb.

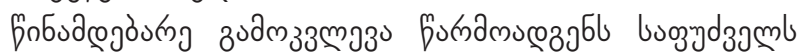

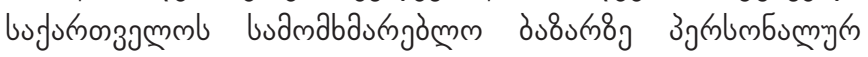

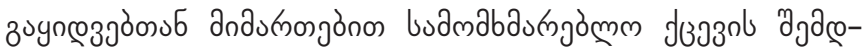

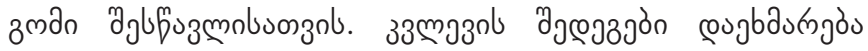

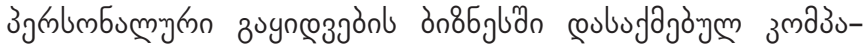

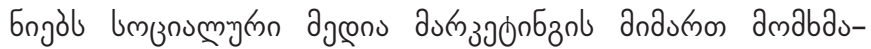

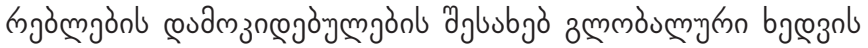

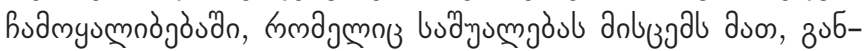

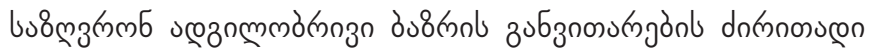

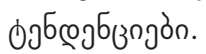

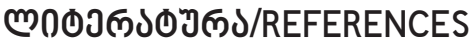

Agnihotri, R., Dingus, R., Hu, M. Y., \& Krush, M. T. (2016). Social media: Influencing customer satisfaction in B2B sales. Industrial Marketing Management, 53, 172-180.

Andzulis, J. M., Panagopoulos, N. G., \& Rapp, A. (2012). A review of social media and implications for the sales process. Journal of Personal Selling \& Sales Management, 32 (3), 305-316.

Apil, A. R., Kaynak, E., \& Todua, N. (2009). Product Preference Differences of High and Low Ethnocentric Consumers in Georgia. In Proceedings of the 18th World Business Congress "Management Challenges in an Environment of Increasing Regional and Global Concerns". International Management Development Association, pp. 531-539.

Ashley, C., \& Tuten, T. (2015). Creative strategies in social media marketing: An exploratory study of branded social content and consumer engagement. Psychology \& Marketing, 32 (1), 15-27.

Atshaya, S., \& Rungta, S. (2016). Digital Marketing vs. Internet Marketing: A Detailed Study. International Journal of Novel Research in Marketing Management and Economics, 3 (1), 29-33.

Avlonitis, G. J., \& Panagopoulos, N. G. (2010). Selling and sales management: An introduction to the special section and recommendations on advancing the sales research agenda. Industrial Marketing Management, 39 (7), 1045-1048.

DataReportal (2020). Digital 2020: Georgia. Produced by Simon Kemp and the team at Kepios [online]. https://datareportal. com/reports/digital-2020-georgia

Hennig-Thurau, T., Malthouse, E. C., Friege, C., Gensler, S., Lobschat, L., Rangaswamy, A., \& Skiera, B. (2010). The impact of new media on customer relationships. Journal of service research, 13 (3), 311-330.

Homburg, C., Wieseke, J., \& Kuehnl, C. (2010). Social influence on salespeople's adoption of sales technology: a multilevel analysis. Journal of the Academy of Marketing Science, 38 (2), 159-168.

Kelly, L., Kerr, G., \& Drennan, J. (2010). Avoidance of advertising in social networking sites: The teenage perspective. Journal of interactive advertising, 10 (2), 16-27.

Kim, A. J., \& Ko, E. (2012). Do social media marketing activities enhance customer equity? An empirical study of luxury fashion brand. Journal of Business research, 65(10), 1480-1486.

Kovac, M. (2016). Social Media Works for B2B Sales, Too. Harvard Business Review (Web Article, Publication Date Jan. 04, 2016).

Malhotra, N. (2004). Marketing Research: An Applied Orientation. 4th ed., Upper Saddle River, NJ: Prentice Hall.

Marshall, G. W., Moncrief, W. C., Rudd, J. M., \& Lee, N. (2012). Revolution in sales: The impact of social media and related technology on the selling environment. Journal of Personal Selling \& Sales Management, 32 (3), 349-363.

Metz, K., \& Hemmann, S. (2011). Making it work: 5 steps to social media success. Public Relations Tactics, 18 (10), 14.

Mghebrishvili, B., \& Urotadze, E. (2016). Functions of Retailing. In Proceedings of 23rd International Academic Conference, Venice, Italy, pp. 356-360.

Minsky, L., \& Quesenberry, K. A. (2016). How B2B Sales can Benefit from Social Selling. Harvard Business Review (Web article. Published on Nov. 08, 2016).

Parveen, F., Jaafar, N. I., \& Ainin, S. (2016). Social media's impact on organizational performance and entrepreneurial orientation in organizations. Management Decision. 54(9), 2208-2234.

Rapp, A., Beitelspacher, L. S., Grewal, D., \& Hughes, D. E. (2013). Understanding social media effects across seller, retailer, and consumer interactions. Journal of the Academy of Marketing Science, 41(5), 547-566.

Richter, A., \& Koch, M. (2007). Social software: Status quo und Zukunft (pp. 1-49). Fak. für Informatik, Univ. der Bundeswehr München. 
Rodriguez, M., Peterson, R. M., \& Krishnan, V. (2012). Social media's influence on business-to-business sales performance. Journal of Personal Selling \& Sales Management, 32 (3), 365-378.

Scott, D. M. (2009). The new rules of marketing and PR: how to use social media, blogs, news releases, online video, and viral marketing to reach buyers directly. John Wiley \& Sons.

Seturi, M., \& Urotadze, E. (2017). About Marketing Process Model and Relationship Marketing. In Proceedings of International Workshop „Model-Based Governance for Smart Organizational Future“; Sapienza University of Rome, Italy, pp. 169-171.

Shin, W., Pang, A., \& Kim, H. J. (2015). Building relationships through integrated online media: Global organizations' use of brand web sites, Facebook, and Twitter. Journal of Business and Technical Communication, 29 (2), 184-220.

Smith, T. (2009). The Social Media Revolution. International Journal of Market Research, 51 (4), 559-561.

Todua, N. (2017). Influence of Food Labeling Awereness on healthy behavior of Georgian Consumers. Ecoforum Journal, 6 (2).

Todua, N. (2017). Social Media Marketing for Promoting Tourism Industry in Georgia. In Proceedings of 22nd International Conference on Corporate and Marketing Communications: Challenges of Marketing Communications in a Globalized World. Zaragoza, Spain, 168-171.

Todua, N. (2019). Attitude of Georgian Consumers to Healthy Nutrition. In Proceedings of 46th International Academic Conference, Rome, Italy, 208-219.

Todua, N., \& Gogitidze, T. (2017). Marketing Research of Attitudes Towards Genetically Modified Crops by Georgian Farmers. Annals of Constantin Brancusi University of Targu-Jiu. Economy Series. (1), 69-76.

Todua, N., \& Dotchviri, T. (2015). Anova in Marketing Research of Consumer Behavior of Different Categories in Georgian Market. Annals of Constantin Brancusi University of Targu-Jiu. Economy Series, (1), 183-190.

Todua, N., \& Jashi, Ch. (2016). Main Aspects of Service Quality in the Hotel Industry of Georgia. In Proceedings of 4th International Conference on Contemporary Marketing Issues (ICCMI). Heraklion, Greece, pp. 431-435.

Todua, N., \& Jashi, Ch. (2018). Influence of Social Marketing on the Behavior of Georgian Consumers Regarding Healthy Nutrition. Bulletin of the Georgian National Academy of Sciences, 12 (2), 183-190.

Todua, N., \& Maghlakelidze, K. (2019). Marketing research on food consumers behavior in Georgiass consumer market. British Journal of Marketing Studies. 7 (4), 76-82.

Todua, N., \& Vashakidze, B. (2018). Consumers Attitudes towards Personal Selling on the Georgian Market. Economics and Business.11 (1). pp. 114-130.

Todua, N., Mghebrishvili, B., \& Urotadze, E. (2016). Main aspects of formation of legal environment on the Georgian food market. In Proceedings of International Scientific-Practical Conference "Challenges of Globalization in Economics and Business". Universal, Tbilisi, pp. 250-256.

Trainor, K. J. (2012). Relating social media technologies to performance: A capabilities-based perspective. Journal of Personal Selling \& Sales Management, 32(3), 317-331.

Urotadze, E. (2020). The role of social media marketing in attitude towards Georgian consumers to travel agencies. In Proceedings of 7th Business Systems Laboratory International Symposium „Socio-Economic Ecosystems: Challenges for Sustainable Development in the Digital Era“. Alicante, Spain, pp. 236-241.

Üstüner, T., \& Godes, D. (2006). Better sales networks. Harvard business review, 84 (7/8), 102-112.

We Are Social, \& Hootsuite (2020). Digital in 2020 [online]. https://wearesocial.com/digital-2020 